\title{
Communication \\ Crystal Structure of the Catalytic Domain of MCR-1 (cMCR-1) in Complex with D-Xylose
}

\author{
Zhao-Xin Liu ${ }^{1,2,+}$, Zhenggang Han ${ }^{1,+}$, Xiao-Li Yu ${ }^{1}$, Guoyuan Wen ${ }^{3}$ and Chi Zeng ${ }^{1,3, * \text { (i) }}$ \\ 1 Hubei Province Engineering Research Center of Healthy Food, School of Biology and Pharmaceutical \\ Engineering, Wuhan Polytechnic University, Wuhan 430023, China; jiangxinklzx@163.com (Z.-X.L.); \\ zhengganghan@whpu.edu.cn (Z.H.); yxll268@126.com (X.-L.Y.) \\ 2 College of Life Sciences, Wuhan University, Wuhan 430072, China \\ 3 Key Laboratory of Prevention and Control Agents for Animal Bacteriosis (Ministry of Agriculture), \\ Institute of Animal Husbandry and Veterinary, Hubei Academy of Agricultural Sciences, Wuhan 430064, \\ China; wgy_524@163.com \\ * Correspondence: czeng@whpu.edu.cn \\ + These authors contributed equally to this work.
}

Received: 4 March 2018; Accepted: 14 April 2018; Published: 17 April 2018

\begin{abstract}
The polymyxin colistin is known as a "last resort" antibacterial drug toward pandrug-resistant enterobacteria. The recently discovered plasmid-encoded $\mathrm{mcr}$ - 1 gene spreads rapidly across pathogenic strains and confers resistance to colistin, which has emerged as a global threat. The $m c r-1$ gene encodes a phosphoethanolamine transferase (MCR-1) that catalyzes the transference of phosphoethanolamine to lipid A moiety of lipopolysaccharide, resulting in resistance to colistin. Development of effective MCR-1 inhibitors is crucial for combating MCR-1-mediated colistin resistance. In this study, MCR-1 catalytic domain (namely cMCR-1) was expressed and co-crystallized together with D-xylose. X-ray crystallographic study at a resolution of $1.8 \AA$ found that cMCR-1-D-xylose co-crystals fell under space group $P 2{ }_{1} 2_{1} 2_{1}$, with unit-cell parameters $a=51.6 \AA$, $b=73.1 \AA, c=82.2 \AA, \alpha=90^{\circ}, \beta=90^{\circ}, \gamma=90^{\circ}$. The asymmetric unit contained a single cMCR-1 molecule complexed with D-xylose and had a solvent content of $29.13 \%$. The structural model of cMCR-1-D-xylose complex showed that a D-xylose molecule bound in the putative lipid A-binding pocket of cMCR-1, which might provide a clue for MCR-1 inhibitor development.
\end{abstract}

Keywords: polymyxin resistance; colistin resistance; MCR-1

\section{Introduction}

Antimicrobial resistance among Gram-negative bacteria, especially the multidrug-resistant enterobacteria which are the leading cause of human clinical infections, is a global healthcare concern [1]. The carbapenemase-producing carbapenem-resistant Enterobacteriaceae (CRE), such as Klebsiella pneumoniae strains expressing the KPC-2 enzyme and Enterobacteriaceae strains expressing the NDM-1 enzyme, are of special clinical importance [1].

Polymyxin is often employed as the final therapeutic option to treat CRE-caused clinical infections because of its low resistance and high efficiency among CRE [2]. Polymyxins (colistin, polymyxin B) are cationic polypeptides which could bind the lipid A moiety of bacterial lipopolysaccharide and disrupt the bacterial cytomembrane subsequently [2]. Bacterial polymyxin resistance was considered to be very low and primarily caused by genomic mutations associated with specific two-component regulatory systems, which either modify lipid A or lead to complete loss of the lipopolysaccharide [2].

Recently, a novel mobile colistin resistance mechanism, led by a protein named MCR-1 (a phosphoethanolamine (PEA) transferase that confers colistin resistance by catalyzing the transference 
of phosphoethanolamine to lipid A moiety of lipopolysaccharide), has been discovered [3]. The gene encoding MCR-1 ( $m c r-1)$ has been shown plasmid-located and self-transmittable between various bacterial strains [2]. Until now, $m c r-1$ has already been detected within a broad range of pathogenic isolates from humans and animals worldwide, which poses a huge threat to the sustaining effectiveness of colistin against CRE-caused clinical infections [2]. Development of effective MCR-1 inhibitors might be the only way to extend the usage of colistin as a reserved antibacterial drug to treat CRE infections [4].

Although several structures of MCR-1 catalytic domain (namely cMCR-1) have been determined [5-8], few effective inhibitors for MCR-1 are known. A recent co-crystallization study [9] showed that two substrate analogues of MCR-1, ethanolamine and D-glucose, could specifically bind to cMCR-1. Here, the crystallization and primary structure analysis of cMCR-1 complexed with D-xylose is reported. The structure determined showed that a D-xylose molecule bound in the putative lipid A-binding pocket of cMCR-1, which might provide a clue for MCR-1 inhibitor development.

\section{Materials and Methods}

\subsection{Recombinant cMCR-1 Production}

The sequence of $m c r-1$ gene is available in GenBank (GenBank accession no. KY685070). Based on the secondary structure predictions, the MCR-1 catalytic domain (namely cMCR-1) includes 326 amino acids, from Pro216 to Arg541. The partial mcr-1 gene sequence encoding cMCR-1 with NcoI/XhoI restriction sites incorporated at the $5^{\prime} / 3^{\prime}$ ends was commercially synthesized and cloned into NcoI/XhoI restriction sites of the expression vector $\mathrm{pET}-28 \mathrm{a}(+)$ (Novagen), creating $\mathrm{pET}-28 \mathrm{a}(+)-\mathrm{mcr}-1$. In construct pET-28a(+)-mcr-1, a histidine tag (HHHHHH) was fused to the C-terminus of cMCR-1 (Table 1).

Table 1. Production specifics for cMCR-1.

\begin{tabular}{|c|c|}
\hline Source & Escherichia coli \\
\hline DNA & Synthesized DNA \\
\hline Forward primer ${ }^{1}$ & 5'-CATGCCATGGCCAAAAGATACCATTTATCAC-3' \\
\hline Reverse primer $^{2}$ & 5'-CCCTCGAGGCGGATGAATGCGGTGCGGTC-3' \\
\hline Expression vector & pET-28a $(+)$ \\
\hline Host & E. coli BL21(DE3)pLysS \\
\hline Recombinant protein sequence ${ }^{3}$ & $\begin{array}{c}\text { MGPKDTIYHAKDAVQATKPDMRKPRLVVF } \\
\text { VVGETARADHVSFNGYERDTFPQLAKIDGVTNF } \\
\text { SNVTSCGTSTAYSVPCMFSYLGADEYDVDTAK } \\
\text { YQENVLDTLDRLGVSILWRDNNSDSKGVMDKLPKA } \\
\text { QFADYKSATNNAICNTNPYNECRDVGMLVGLDDFV } \\
\text { AANNGKDMLIMLHQMGNHGPAYFKRYDEKFAKFT } \\
\text { PVCEGNELAKCEHQSLINAYDNALLATDDFIAQSIQ } \\
\text { WLQTHSNAYDVSMLYVSDHGESLGENGVYLHGMP } \\
\text { NAFAPKEQRSVPAFFWTDKQTGITPMATDTVLTHD } \\
\text { AITPTLLKLFDVTADKVKDRTAFIRLEHHHHHH }\end{array}$ \\
\hline
\end{tabular}

${ }^{1}$ The NcoI site noted. ${ }^{2}$ The XhoI site noted. ${ }^{3}$ The cloning artifacts are underlined.

Escherichia coli BL21(DE3)pLysS was transformed with pET-28a(+)-mcr-1 and grown at $310 \mathrm{~K}$, $200 \mathrm{rpm}$ rotation in LB liquid medium containing $50 \mu \mathrm{g} \mathrm{mL}{ }^{-1}$ kanamycin for cMCR-1 expression. Confluent cultures $\left(\mathrm{OD}_{600} \sim 0.6\right)$ were then treated with $0.3 \mathrm{mM}$ (final concentration) IPTG at $298 \mathrm{~K}$ with shaking $(180 \mathrm{rpm})$ for $20 \mathrm{~h}$. Cells were collected by $20 \mathrm{~min}$ of centrifugation $(4500 \mathrm{~g}, 277 \mathrm{~K})$ and pellets were kept at $193 \mathrm{~K}$ for subsequent use.

The cell pellets were lysed with $10 \mathrm{mM}$ Tris- $\mathrm{HCl} \mathrm{pH}$ 8.0, $200 \mathrm{mM} \mathrm{NaCl}, 5 \%$ (v/v) glycerol, 0.3\% $(v / v)$ Triton X-100, $1 \mathrm{mM}$ DTT, and $0.1 \mathrm{mM}$ PMSF in a French press. Cell wastes were excluded by 
centrifuging the lysates at $12,000 \mathrm{~g}$ for $30 \mathrm{~min}$ at $277 \mathrm{~K}$, and the supernatant was clarified using a $0.45 \mu \mathrm{m}$ filter and then passed through a pre-equilibrated Ni-NTA affinity column (GE Healthcare). The affinity column was washed thoroughly using $10 \mathrm{mM}$ Tris- $\mathrm{HCl} \mathrm{pH} 8.0$ containing $50 \mathrm{mM}$ imidazole to remove the miscellaneous proteins. The target proteins were eluted using $10 \mathrm{mM}$ Tris- $\mathrm{HCl} \mathrm{pH} 8.0$ containing $200 \mathrm{mM}$ imidazole. Concentrated protein was then loaded onto a MonoQ 5/50 GL anion exchange column (GE Healthcare) and chromatographed at $1 \mathrm{~mL} \mathrm{~min}^{-1}$ using a linear $\mathrm{NaCl}$ gradient generated with $10 \mathrm{mM}$ Tris- $\mathrm{HCl} \mathrm{pH} 8.0$ (buffer A) and $10 \mathrm{mM}$ Tris- $\mathrm{HCl}$ pH 8.0, $1 \mathrm{M} \mathrm{NaCl}$ (buffer B). Peak fractions were pooled and run through a Superdex 200 10/300 GL column (GE Healthcare) equilibrated with buffer $\mathrm{A}$ at a flow rate of $1 \mathrm{~mL} \mathrm{~min}^{-1}$. Peak fractions were recovered and concentrated to $10 \mathrm{mg}$ $\mathrm{mL}^{-1}$ for crystallization. The purity of the final protein (cMCR-1) was checked by SDS-PAGE. All specifics for recombinant cMCR-1 production are present in Table 1.

\subsection{Crystallization}

The cMCR-1 was crystallized at $277 \mathrm{~K}$ using the sitting-drop vapor-diffusion method as described by Wei et al. [9]. The $0.5 \mu \mathrm{L}$ sitting drops consisting of $0.25 \mu \mathrm{L}$ cMCR-1 solution and $0.25 \mu \mathrm{L}$ reservoir solution were equilibrated against $30 \mu \mathrm{L}$ reservoir solution in 96-well MRC plates (Molecular Dimensions). The best crystals were achieved in 10\% (w/v) PEG 1000, 5\% (w/v) PEG 8000.

\subsection{Data Collection, Structure Solution, and Refinement}

The cMCR-1 crystals were incubated in mother liquor containing $100 \mathrm{mM}$ D-xylose for $10 \mathrm{~s}$ to form the cMCR-1-D-xylose complex. The cMCR-1-D-xylose co-crystals used for diffraction data collection were incubated in cryoprotectant (mother liquor containing 20\% $(v / v)$ glycerol) for $10 \mathrm{~s}$ before flash cooling in streams of liquid nitrogen. Data for CMCR-1-D-xylose complex were acquired at $100 \mathrm{~K}$ using an ADSC Q315r detector at beamline BL17U1 of Shanghai Synchrotron Radiation Facility (SSRF), China; 360 frames were taken with $1.0^{\circ}$ oscillations. The data were indexed, integrated, and scaled using HKL-2000 (HKL Research, Inc., Charlottesville, VA, USA) [10] and iMosflm programs [11]. The structure was solved by molecular replacement with Phaser [12] using a single monomer of cMCR-1 (PDB entry 5GRR [7]) as the search model. The structure model was constructed using alternating manual building in Coot [13] and restrained refinement in PHENIX [14]. The final model was optimized on PDB_REDO web server [15] and validated by MolProbity [16]. All figures were prepared by PyMOL (Schrödinger). Table 2 summarizes data-collection and crystallographic statistics of cMCR-1-D-xylose complex. Coordinates and structure factors of the CMCR-1-D-xylose complex have been deposited in the Protein Data Bank (PDB) under accession code 5ZJV. 
Table 2. Data-collection and crystallographic statistics of cMCR-1-D-xylose complex.

\begin{tabular}{|c|c|}
\hline Diffraction Source & BL17U1, SSRF \\
\hline Wavelength $(\AA)$ & 0.9792 \\
\hline Temperature (K) & 100 \\
\hline Detector & ADSC Q315r \\
\hline Crystal-to-detector distance (mm) & 350 \\
\hline Total rotation range $\left({ }^{\circ}\right)$ & 360 \\
\hline Rotation range per image $\left(^{\circ}\right)$ & 1.0 \\
\hline Exposure time per image (s) & 0.5 \\
\hline Space group & $P 2_{1} 2_{1} 2_{1}$ \\
\hline$a, b, c(\AA)$ & $51.6,73.1,82.2$ \\
\hline$\alpha, \beta, \gamma\left({ }^{\circ}\right)$ & $90,90,90$ \\
\hline Resolution range $(\AA)$ & $43.71-1.82(1.88-1.82)^{1}$ \\
\hline Total number of reflections & $56014(5514)$ \\
\hline Number of unique reflections & $28258(2789)$ \\
\hline Mosaicity $\left(^{\circ}\right)$ & 0.5 \\
\hline Multiplicity & $2.0(2.0)$ \\
\hline Completeness (\%) & $99.0(99.0)$ \\
\hline Mean $I / \sigma(I)$ & $10.61(4.94)$ \\
\hline$R_{\text {merge }}(\%)$ & $3.5(11.1)$ \\
\hline $\mathrm{CC}_{1 / 2}$ & $0.997(0.949)$ \\
\hline Wilson plot overall $B$ factor $\left(\AA^{2}\right)$ & 13.74 \\
\hline Reflection number, working set & $28223(2785)$ \\
\hline Reflection number, test set & $1997(196)$ \\
\hline$R_{\text {work }}$ & 0.139 \\
\hline$R_{\text {free }}$ & 0.178 \\
\hline Ramachandran favored region (\%) & 98 \\
\hline Ramachandran allowed region (\%) & 1.75 \\
\hline Ramachandran outliers (\%) & 0.25 \\
\hline Rotamer outliers (\%) & 0.65 \\
\hline R.m.s.d. bond lengths $(\AA)$ & 0.006 \\
\hline R.m.s.d. bond angles $\left({ }^{\circ}\right)$ & 0.86 \\
\hline Average $B$ factor $\left(\AA^{2}\right)$ & 17.55 \\
\hline
\end{tabular}

${ }^{1}$ Outer shell values.

\section{Results and Discussion}

As stated in the Introduction, development of effective MCR-1 inhibitors is crucial for combating the threat of colistin resistance mediated by MCR-1. A recent co-crystallization study [9] showed that two substrate analogues of MCR-1 (ethanolamine and D-glucose) could specifically bind to MCR-1 catalytic domain (cMCR-1). Both D-glucose and lipid A are hexacyclic compounds. Thus, we tried many other hexacyclic compounds for co-crystallization with cMCR-1 (unpublished). The only other co-crystal structure was that obtained for the complex formed between CMCR-1 and D-xylose at a resolution of $1.8 \AA$.

MCR-1 belongs to the phosphoethanolamine (PEA) transferase family. It contains 541 amino acids with an N-terminal five-helix transmembrane domain (amino acid residues 1-215) and a C-terminal periplasmic catalytic domain (amino acid residues 216-541) [9]. In order to investigate the potential interactions between D-xylose (and other hexacyclic compounds) and MCR-1, cMCR-1 (MCR-1 catalytic domain) was expressed and purified using a combine of affinity, anion exchange and gel filtration chromatography (Figure 1a), as stated in Section 2.1. The purity of the purified cMCR-1 was confirmed with SDS-PAGE (Figure 1b) and subsequent Western blot analysis (Figure 1c). The cMCR-1 was effectively crystallized using the sitting-drop vapor-diffusion method as described by Wei et al. [9], which generated diffraction-quality crystals with a longest dimension of $0.2 \mathrm{~mm}$ (Figure 2). SDS-PAGE showed that the obtained crystals were protein crystals and had the same molecular weight as the 
purified cMCR-1 protein (Figure 1b). The cMCR-1 crystals were incubated in the mother liquor supplemented with $100 \mathrm{mM}$ D-xylose for $10 \mathrm{~s}$ to form the cMCR-1-D-xylose complex.

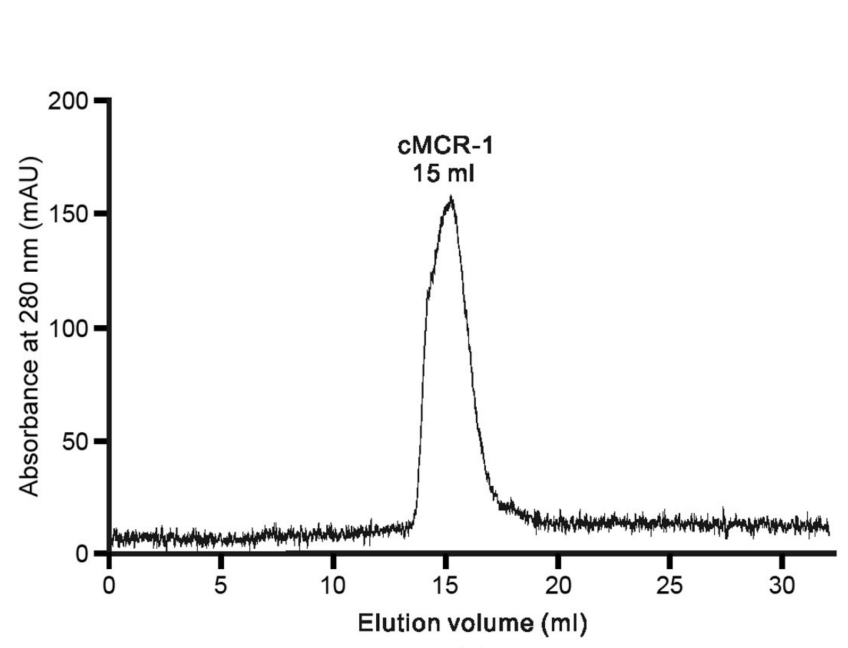

(a)

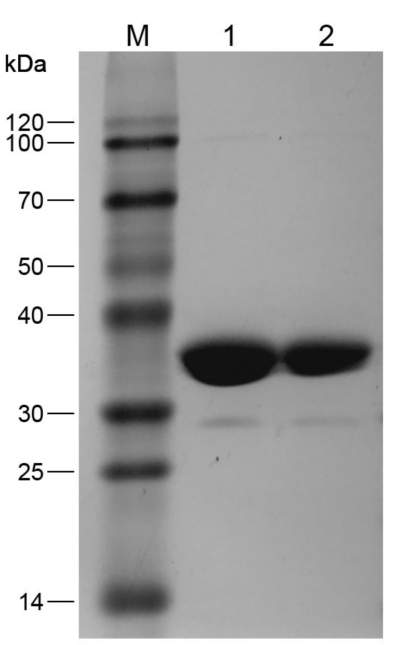

(b)

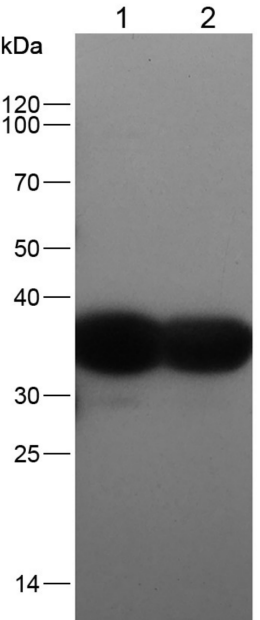

(c)

Figure 1. Purification and purity analysis of cMCR-1. (a) Gel filtration chromatography of cMCR-1. (b) SDS-PAGE of the final purified cMCR-1 and cMCR-1 crystals. Molecular-weight markers (lane M, labelled in $\mathrm{kDa}$ ), purified $\sim 35 \mathrm{kDa}$ cMCR-1 protein (lane 1) and cMCR-1 crystals (lane 2) are shown. (c) Western blot analysis of the final purified cMCR- 1 and cMCR- 1 crystals using an anti- $6 \times$ His antibody. Purified $~ 35$ kDa cMCR-1 protein (lane 1) and cMCR-1 crystals (lane 2) are shown.

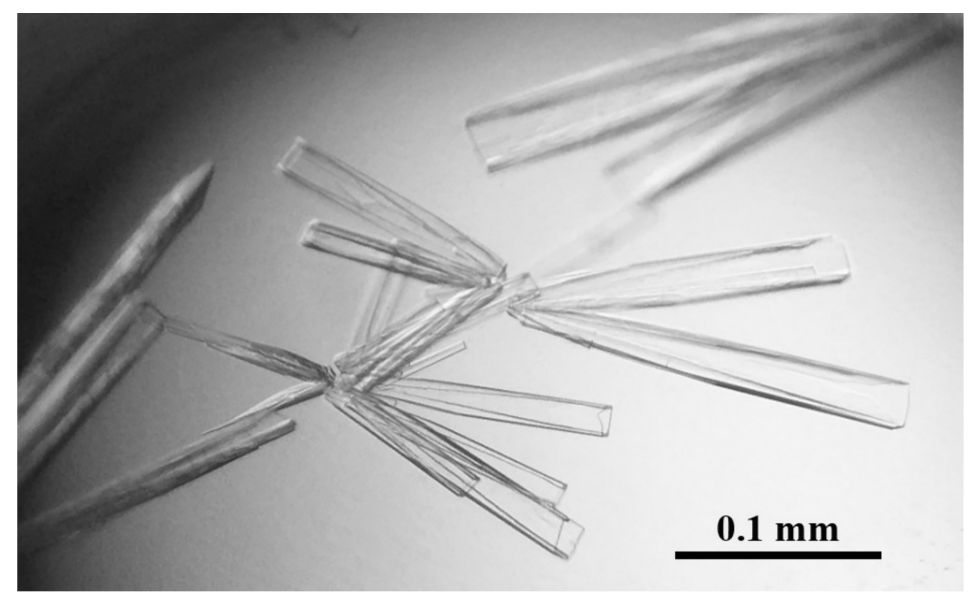

Figure 2. Crystals of cMCR-1.

Diffraction data for the cMCR-1-D-xylose complex was collected to $1.8 \AA$ resolution (Figure 3) and on its basis, the cMCR-1-D-xylose co-crystals fell under space group $P 2{ }_{1} 2_{1} 2_{1}$, possessing unit-cell parameters $a=51.6 \AA, b=73.1 \AA, c=82.2 \AA, \alpha=90^{\circ}, \beta=90^{\circ}, \gamma=90^{\circ}$. The asymmetric unit contained a single cMCR-1 molecule complexed with D-xylose. The data set of X-ray diffraction had a resolution range from $43.71 \AA$ to $1.82 \AA$ with $3.5 \% R_{\text {merge }}$ and $99.0 \%$ completeness. To elucidate the structure of cMCR-1-D-xylose complex, we employed molecular replacement method using cMCR-1 monomer (PDB entry 5GRR [7]) as a search model and obtained a clear solution. We confirmed the occurrence of a single protein molecule in the asymmetric unit by cross-rotation and translation-function calculations; the corresponding solvent content was $29.13 \%$. Initial structure refinement using PHENIX [14] yielded a model (Figure 4 a) with an $R_{\text {work }}$ of $13.9 \%$ and an $R_{\text {free }}$ of $17.8 \%$. 


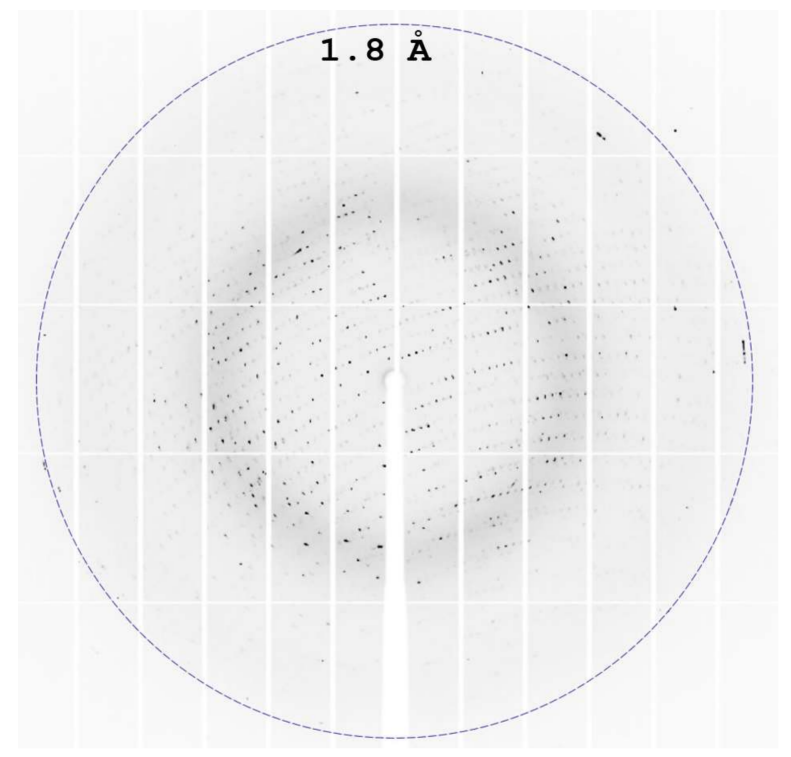

Figure 3. Representative X-ray diffraction pattern of cMCR-1-D-xylose co-crystal.

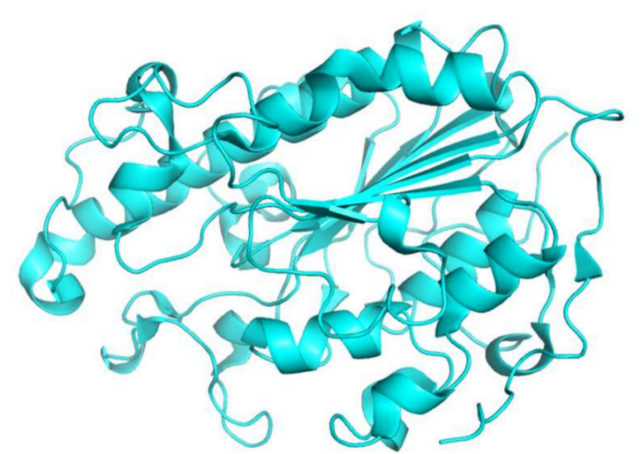

(a)

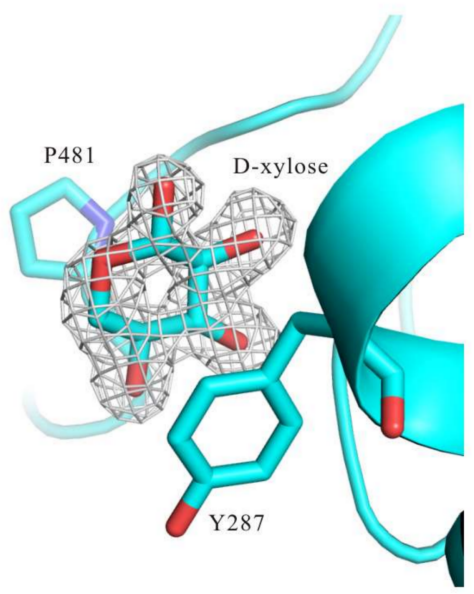

(c)

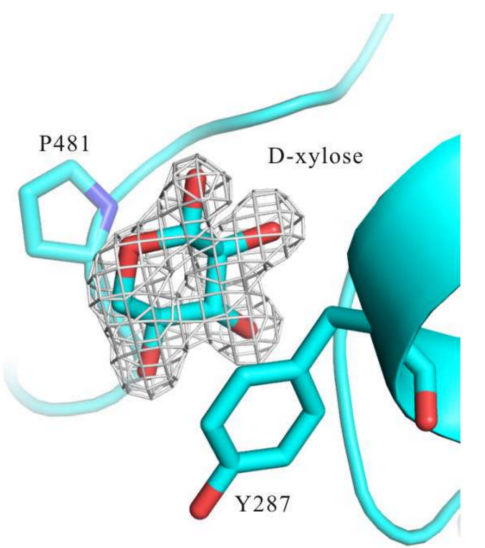

(b)

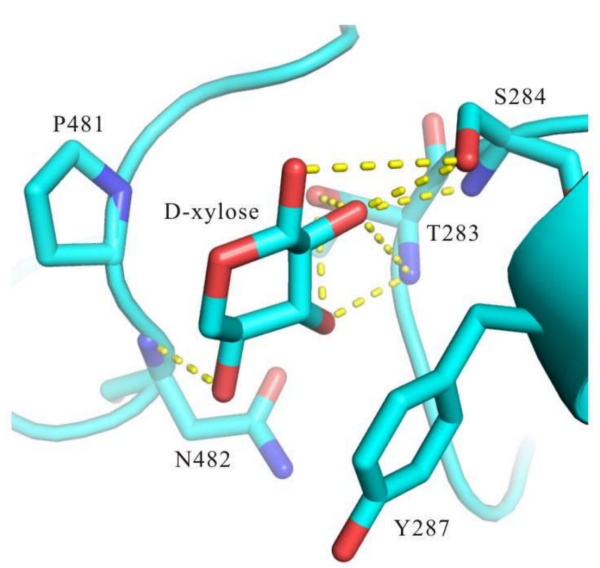

(d)

Figure 4. Cont. 


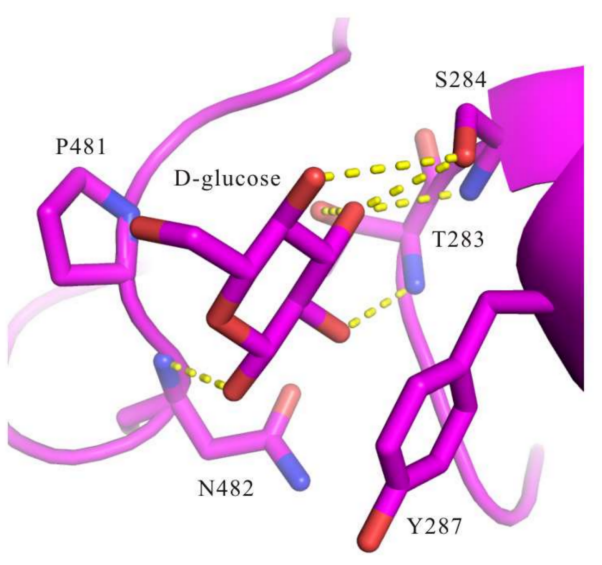

(e)

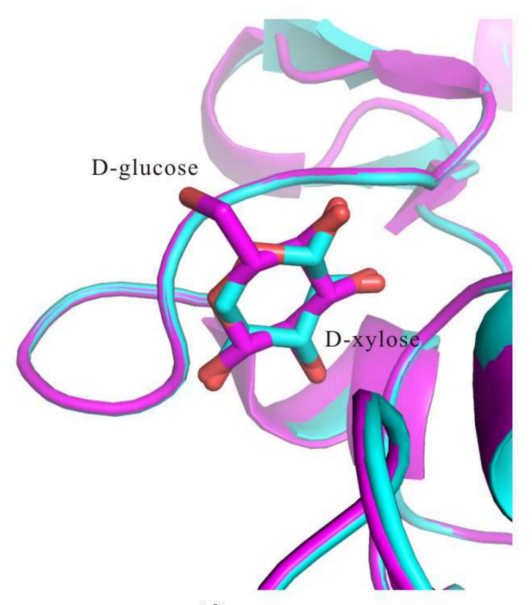

(f)

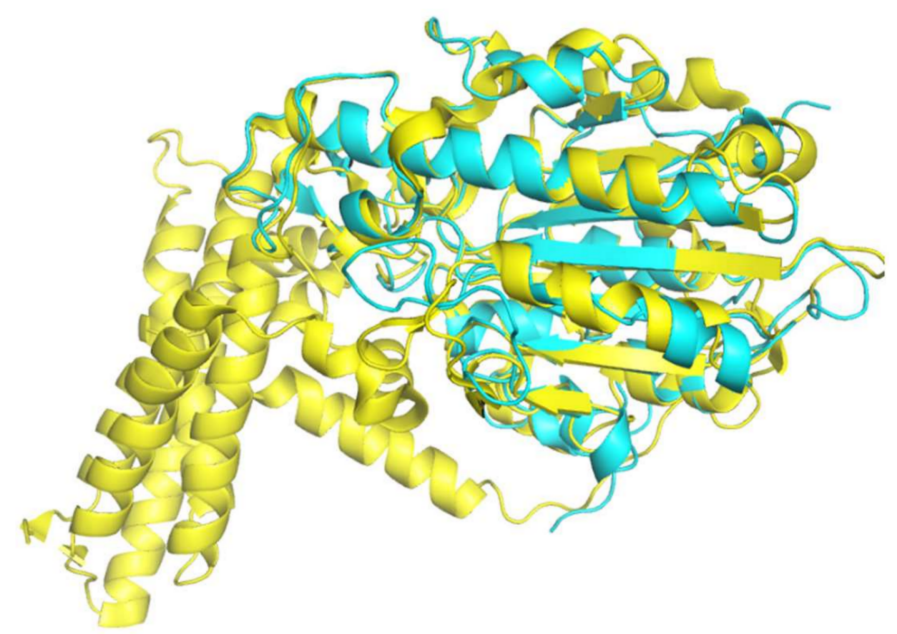

(g)

Figure 4. Structure of the cMCR-1-D-xylose complex. (a) The ribbon diagram showing the overall structure of cMCR-1. (b) The Fo-Fc electron-density map contoured at $3.0 \sigma$ depicting the D-xylose molecule. The map was calculated using the model omitting the D-xylose molecule after rounds of refinement. (c) The 2Fo-Fc electron-density map contoured at $1.0 \sigma$ depicting the D-xylose molecule. (d) Interaction between cMCR-1 and the D-xylose molecule. (e) Interaction between cMCR-1 and the D-glucose molecule. (f) Superposition of cMCR-1-D-xylose (cyan) and cMCR-1-D-glucose (magenta). (g) Superposition of cMCR-1-D-xylose (cyan) and EptA (yellow). All figures were prepared using PyMOL (Schrödinger).

As shown in the Fo-Fc map (contoured at $3.0 \sigma$ level) (Figure 4b) and 2Fo-Fc map (contoured at $1.0 \sigma$ level) (Figure 4c), a D-xylose molecule bound in the putative lipid A-binding pocket of cMCR-1. According to the PDB structure validation report, real space correlation coefficient (RSCC) and real space r-value (RSR) are 0.94 and 0.13, respectively, for the ligand D-xylose. The D-xylose, Pro481, and Tyr287 formed a sandwich structure with the D-xylose molecule in the middle (Figure 4d). Obviously, the hydrophobic stacking interaction played a crucial role in D-xylose recognition.

The structure of cMCR-1-D-xylose complex is similar with that of cMCR-1-D-glucose complex (PDB entry 5YLF [9]). Meanwhile, there are still many differences between the two structures. First, alignment of the two structures (Figure 4f) showed that both D-xylose and D-glucose bound to the same pocket of cMCR-1 and formed a $\pi$ - $\pi$-conjugated interaction with Pro481 and Tyr287 of cMCR-1, but the skeletons of D-xylose and D-glucose were in the opposite positions. Next, we analyzed the cMCR-1-D-xylose and cMCR-1-D-glucose interactions by using 
PISA server (http://www.ebi.ac.uk/pdbe/pisa). The accessible surface area (ASA) $=269.67 \AA^{2}$, buried surface area $(B S A)=194.27 \AA^{2}$, solvation energy effect $\left(\Delta^{\mathrm{i}} G\right)=-2.37 \mathrm{kcal} / \mathrm{mol}$ between D-xylose and cMCR-1, and the ASA $=303.17 \AA^{2}, B S A=204.82 \AA^{2}, \Delta^{\mathrm{i}} \mathrm{G}=-2.97 \mathrm{kcal} / \mathrm{mol}$ between D-glucose and cMCR-1. It suggested that the interaction between D-glucose and cMCR-1 is greater than that between D-xylose and CMCR-1. The D-xylose and D-glucose molecules also bound to cMCR-1 through a large number of hydrogen bonds. The O1, O2, O3, and O4 atoms of D-xylose hydrogen-bonded to Ser284 OG, Thr283 OG1, N/Ser284 N, OG, Thr283 OG1, N, and Asn482 N, respectively (Figure 4d). The O1, O2, O3, and O4 atoms of D-glucose hydrogen-bonded to Asn482 N, Thr283 N, Ser284 N, OG/Thr283 OG1, and Ser284 OG, respectively (Figure 4e).

We also conducted a comparison of cMCR-1-D-xylose complex with phosphoethanolamine transferase A (EptA) from Neisseria meningitides (PDB entry 5FGN [17]), the only structure of a full-length phosphoethanolamine transferase so far. The structure of cMCR-1-D-xylose complex can be well superimposed with the structure of EptA catalytic domain, with a C $\alpha$ root-mean-square deviation of $2.0 \AA$ as revealed by Dali server (http://ekhidna.biocenter.helsinki.fi/dali_server/start) [18] (Figure 4g). Anandan et al. [17] have shown that detergent dodecyl- $\beta$-D-maltoside (DDM) could bind in a substrate pocket of EptA, and the pocket bound by DDM was probably the phosphoethanolamine (PEA) binding pocket near the putative lipid A-binding pocket.

In conclusion, our finding that D-xylose could bind in the putative lipid A-binding pocket of cMCR-1 is interesting, which might provide a clue for MCR-1 inhibitor development. In vitro inhibitory assay is currently in progress to confirm if D-xylose could inhibit colistin resistance mediated by MCR-1.

Acknowledgments: Our study has been aided by an open grant from the Key Laboratory of Prevention and Control Agents for Animal Bacteriosis (Ministry of Agriculture) (KLAEMB-2017-07), a grant from the Research and Innovation Initiatives of WHPU (2018Y11), and National Science and Technology Major Project (2017ZX10201301-003-003).

Author Contributions: Zhao-Xin Liu and Chi Zeng designed the experiments. Zhao-Xin Liu performed the experiments. Zhao-Xin Liu, Chi Zeng, and Zhenggang Han analyzed the data. Xiao-Li Yu and Guoyuan Wen provided technical support. Zhao-Xin Liu, Chi Zeng, and Zhenggang Han completed the paper.

Conflicts of Interest: No interest conflict exists among the authors.

\section{References}

1. Logan, L.K.; Weinstein, R.A. The epidemiology of carbapenem-resistant Enterobacteriaceae: The impact and evolution of a global menace. J. Infect. Dis. 2017, 215, S28-S36. [CrossRef] [PubMed]

2. Poirel, L.; Jayol, A.; Nordmann, P. Polymyxins: Antibacterial activity, susceptibility testing, and resistance mechanisms encoded by plasmids or chromosomes. Clin. Microbiol. Rev. 2017, 30, 557-596. [CrossRef] [PubMed]

3. Liu, Y.Y.; Wang, Y.; Walsh, T.R.; Yi, L.X.; Zhang, R.; Spencer, J.; Doi, Y.; Tian, G.; Dong, B.; Huang, X.; et al. Emergence of plasmid-mediated colistin resistance mechanism MCR-1 in animals and human beings in China: A microbiological and molecular biological study. Lancet Infect. Dis. 2016, 16, 161-168. [CrossRef]

4. Hu, Y.Y.; Wang, Y.L.; Sun, Q.L.; Huang, Z.X.; Wang, H.Y.; Zhang, R.; Chen, G.X. Colistin resistance gene mor-1 in gut flora of children. Int. J. Antimicrob. Agents 2017, 50, 593-597. [CrossRef] [PubMed]

5. Hinchliffe, P.; Yang, Q.E.; Portal, E.; Young, T.; Li, H.; Tooke, C.L.; Carvalho, M.J.; Paterson, N.G.; Brem, J.; Niumsup, P.R.; et al. Insights into the mechanistic basis of plasmid-mediated colistin resistance from crystal structures of the catalytic domain of MCR-1. Sci. Rep. 2017, 7, 39392. [CrossRef] [PubMed]

6. Hu, M.; Guo, J.; Cheng, Q.; Yang, Z.; Chan, E.W.C.; Chen, S.; Hao, Q. Crystal structure of Escherichia coli originated MCR-1, a phosphoethanolamine transferase for colistin resistance. Sci. Rep. 2016, 6, 38793. [CrossRef] [PubMed]

7. Ma, G.; Zhu, Y.; Yu, Z.; Ahmad, A.; Zhang, H. High resolution crystal structure of the catalytic domain of MCR-1. Sci. Rep. 2016, 6, 39540. [CrossRef] [PubMed]

8. Stojanoski, V.; Sankaran, B.; Prasad, B.V.; Poirel, L.; Nordmann, P.; Palzkill, T. Structure of the catalytic domain of the colistin resistance enzyme MCR-1. BMC Biol. 2016, 14, 81. [CrossRef] [PubMed] 
9. Wei, P.; Song, G.; Shi, M.; Zhou, Y.; Liu, Y.; Lei, J.; Chen, P.; Yin, L. Substrate analog interaction with MCR-1 offers insight into the rising threat of the plasmid-mediated transferable colistin resistance. FASEB J. 2018, 32, 1085-1098. [CrossRef] [PubMed]

10. Otwinowski, Z.; Minor, W. Processing of X-ray diffraction data collected in oscillation mode. Methods Enzymol. 1997, 276, 307-326. [CrossRef] [PubMed]

11. Battye, T.G.; Kontogiannis, L.; Johnson, O.; Powell, H.R.; Leslie, A.G. iMOSFLM: A new graphical interface for diffraction-image processing with MOSFLM. Acta Crystallogr. D Biol. Crystallogr. 2011, 67, 271-281. [CrossRef] [PubMed]

12. McCoy, A.J.; Grosse-Kunstleve, R.W.; Adams, P.D.; Winn, M.D.; Storoni, L.C.; Read, R.J. Phaser crystallographic software. J. Appl. Crystallogr. 2007, 40, 658-674. [CrossRef] [PubMed]

13. Emsley, P.; Cowtan, K. Coot: Model-building tools for molecular graphics. Acta Crystallogr. D Biol. Crystallogr. 2004, 60, 2126-2132. [CrossRef] [PubMed]

14. Adams, P.D.; Afonine, P.V.; Bunkóczi, G.; Chen, V.B.; Davis, I.W.; Echols, N.; Headd, J.J.; Hung, L.W.; Kapral, G.J.; Grosse-Kunstleve, R.W.; et al. PHENIX: A comprehensive Python-based system for macromolecular structure solution. Acta Crystallogr. D Biol. Crystallogr. 2010, 66, 213-221. [CrossRef] [PubMed]

15. Joosten, R.P.; Long, F.; Murshudov, G.N.; Perrakis, A. The PDB_REDO server for macromolecular structure model optimization. IUCrJ 2014, 1, 213-220. [CrossRef] [PubMed]

16. Chen, V.B.; Arendall, W.B.; Headd, J.J.; Keedy, D.A.; Immormino, R.M.; Kapral, G.J.; Murray, L.W.; Richardson, J.S.; Richardson, D.C. MolProbity: All-atom structure validation for macromolecular crystallography. Acta Crystallogr. D Biol. Crystallogr. 2010, 66, 12-21. [CrossRef] [PubMed]

17. Anandan, A.; Evans, G.L.; Condic-Jurkic, K.; O’Mara, M.L.; John, C.M.; Phillips, N.J.; Jarvis, G.A.; Wills, S.S.; Stubbs, K.A.; Moraes, I.; et al. Structure of a lipid A phosphoethanolamine transferase suggests how conformational changes govern substrate binding. Proc. Natl. Acad. Sci. USA 2017, 114, 2218-2223. [CrossRef] [PubMed]

18. Holm, L.; Laakso, L.M. Dali server update. Nucleic Acids Res. 2016, 44, W351-W355. [CrossRef] [PubMed]

(C) 2018 by the authors. Licensee MDPI, Basel, Switzerland. This article is an open access article distributed under the terms and conditions of the Creative Commons Attribution (CC BY) license (http:/ / creativecommons.org/licenses/by/4.0/). 